\title{
A waveguide based microfluidic application
}

\author{
Nooshin S. Taheri ${ }^{1 \mathrm{a}}$, Peggy Chan ${ }^{1}$, James R. Friend ${ }^{1}$, Leslie Yeo ${ }^{1}$, \\ ${ }^{1}$ Micro/Nano Physics Research Laboratory (MNRL), School of Electrical and Computer Science, RMIT \\ University, Melbourne, Australia \\ ${ }^{a}$ Contact author,s3418889@student.rmit.edu.au
}

\begin{abstract}
Microfluidics is based on the performance of fluids in a microenvironment. As the microfluidics research advances in the cellular behaviour, the need for improved micro devices grows. This work introduces the design and fabrication of a micro ridge waveguide to be employed in fluids manipulations. Then it investigates the characteristics of the device in order to control the movement of the fluids on top of the ridge of the waveguide. The elastic vibration is excited along the ridge of the guide with the use of thickness poled lead zirconate titanate (PZT) elements attached to both sides of the waveguide. To excite anti-symmetric or flexural mode in the ridge of the guide, the propagation velocity has been kept significantly below the Rayleigh wave velocity. The velocity reduction of $15 \%$ is achieved with the high aspect ratio ridge $(\mathrm{H} / \mathrm{W}=3)$ design. A three dimensional model of the micro waveguide has also been developed to determine the vibration characteristics; the natural frequency and the considered mode of the micro waveguide through finite element analysis using ANSYS. The travelling wave along the ridge of the guide is able to transmit strong vibration to the fluid atop of the substrate. The results represents a promising approach, through recasting the waveguide structure to be suitable in fluids and particle in fluids manipulations in one dimensional environment with the strong confined energy, at smaller scale with higher vibration displacement.
\end{abstract}

Key words: acoustic waveguide, micro-scale waveguide, flexural mode, piezoelectric materials, finite element analysis, microfluidics.

\section{INTRODUCTION}

Waveguide structures have been widely studied for acoustic surface waves on their various types and properties (Oliner, 1976) ${ }^{1}$. The guiding, in topographic waveguides, is achieved by deformation of the substrate surface. The lateral extent of the surface wave is confined within the structure's deformation. The wave bound to the waveguide will also smoothly follow any waveguide bends, as long as the radius of curvature of the bend is not too small. The way to interface this with microfluidics is to alter the waveguide structure to form a unique shape for the applications in fluid manipulations.

This research emphasizes on reliably make the waveguide structure micro scale. The next part is to recast the waveguide structure to form a distinctive configuration with best performance regarding fluid behaviour. Last

Micro/Nano Materials, Devices, and Systems, edited by James Friend, H. Hoe Tan, Proc. of SPIE

Vol. 8923, 89234O · C 2013 SPIE · CCC code: 0277-786X/13/\$18 · doi: 10.1117/12.2033743

Proc. of SPIE Vol. $8923892340-1$ 
integrating the proper waveguide structure into microfluidic chip for applications like, flow generation, drop in a fluid or particle in a fluid transport.

This paper presents the results of downsizing acoustic waveguide to micro scale, along with its vibration characteristics.

\section{METHODS}

Figure 1, illustrates the simple outline of the ridge waveguide with the piezoelectric elements (PZT 5-H) attached to the waveguide sides. The ridge cross section is designed to excite only anti-symmetric flexural mode at $986 \mathrm{kHz}$ frequency with a reference to Lagasse's ${ }^{2}$ (Lagasse and Mason, 1972) work. The fine ridge with such a rectangular cross section is machined from aluminium by means of micro machining. The width and the height of the ridge are $200 \mu \mathrm{m}$ and $600 \mu \mathrm{m}$ respectively.

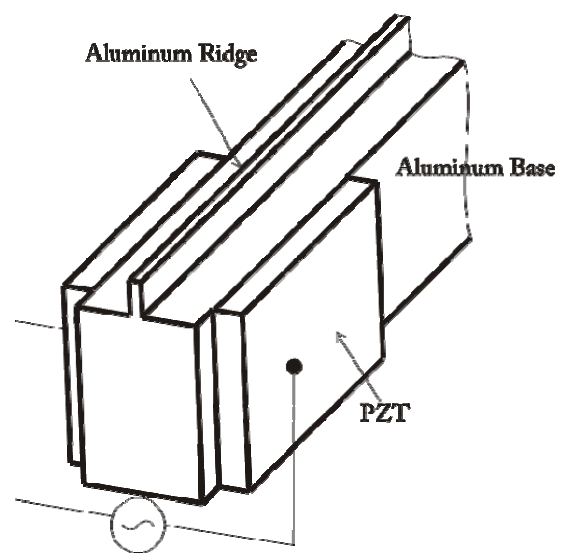

Figure 1. The outline of micro waveguide with attached PZT elements on both sides.

Two piezoelectric ceramic elements are attached to both side of the base of the waveguide with the distance of a wavelength from the end of the base. To monitor the vibration displacement of the device, a slider was placed on top of the ridge waveguide. To excite anti-symmetric or flexural mode in the ridge, the propagation velocity has been kept significantly below the Rayleigh wave velocity. The velocity reduction of $15 \%$ is achieved with the high aspect ratio ridge $(\mathrm{H} / \mathrm{W}=3)$ design. At the frequency of $986 \mathrm{kHz}$ the flexural mode is excited according to the ridge mode's dispersion characteristics from Laggase et $\mathrm{al}^{2}$, as shown in Figure 2. 


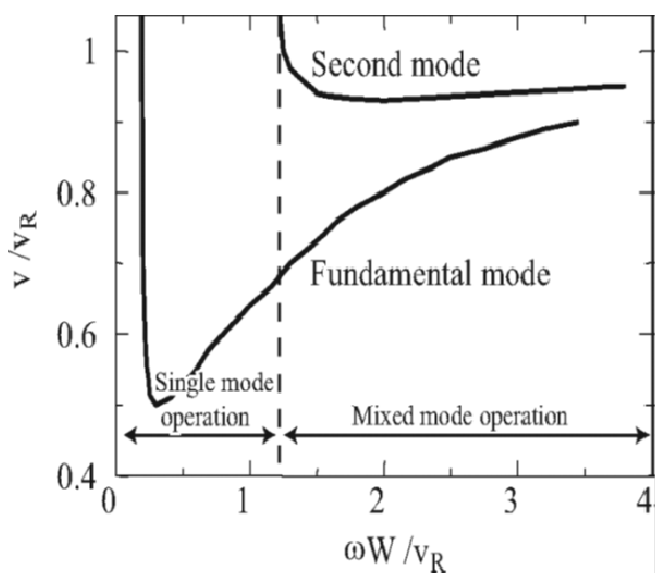

Figure 2. The dispersion characteristics of the waveguide taken from Laggase ${ }^{2}$.

Also a three dimensional model of the micro waveguide was developed in SolidWorks. Finite element analysis was employed using ANSYS (Workbench 14.5) software. Modal analysis was carried out to investigate different modes of vibration in the waveguide.

\section{RESULTS}

The aluminium waveguide, with the ridge dimensions of $200-\mu \mathrm{m}$ wide, $600-\mu \mathrm{m}$ tall, was piezoelectrically excited with an absorbent material at its opposite end to achieve traveling wave. The damping material acts as a terminator for the propagating wave.

The vibration velocity of the side edge of the ridge was measured using laser Doppler vibrometer (MSA-500). The waveguide's vibration velocity was measured to be $2.55 \mathrm{~mm} / \mathrm{s}$.

Modal Analysis presented a total of 14 different mode shapes within the frequency range of $900-1000 \mathrm{kHz}$ for the micro waveguide structure. A couple of mode shapes at the resonance frequencies of $972 \mathrm{kHz}$ and $976 \mathrm{kHz}$ had the desired vibration patterns.

The flexural mode was only excited within the waveguide ridge at $994 \mathrm{kHz}$, the PZT elements were driven at resonance at the same frequency, applying $55 \mathrm{~V}_{\mathrm{o}-\mathrm{p}}$ to the PZT elements. The c-shaped slider was 5-wavelengthlong copper foil to be placed atop of the ridge. The sliding motion was observed at resonance frequency, with the applied voltage of $47.5 \mathrm{~V}_{0-\mathrm{p}}$ to the PZT elements. The maximum velocity of the moving slider towards the source of energy was measured to be about $2.4 \mathrm{~mm} / \mathrm{s}$, using Laser Doppler velocity sensor (LV-20Z).

\section{CONCLUSION}

The acoustic waveguide is a simple linear micro motor that could be integrated into a microfluidic chip towards its applications. With downsizing the acoustic waveguide to micro scale, a promising candidate for microfluidics application is constructed. Generation of vibration in the rectangular waveguide structure and 
transmission of traveling wave in the aluminium waveguide using an absorbing material was presented. The micro waveguide was shown to move the slider in fairly small-scale model. These results pave the way to recasting micro waveguide structures with better configurations and performances for flow generation, particle in fluid transportation applications.

\section{REFERENCE}

[1] OLINER, A. A. 1976. WAVEGUIDES FOR ACOUSTIC SURFACE-WAVES - REVIEW. Proceedings of the Ieee, 64, 615-627.

[2] LAGASSE, P. E. \& MASON, I. M. 1972. ANALYSIS OF PROPAGATION IN RIDGE GUIDES FOR ACOUSTIC SURFACE-WAVES. Electronics Letters, 8, 82-\&. 